\title{
Variação cromossômica em Cactáceas
}

\author{
Chromosomal variation of Cacti
}

\author{
José Geraldo de Aquino Assis ${ }^{* 1,2}$, Juliana Fraga Vasconcelos Senra ${ }^{3}$, Roberta Borges Botelho, \\ Alberto Bispo dos Santos ${ }^{5}$ e Alessandra Selbach Schnadelbach ${ }^{1,2}$ \\ ${ }^{1}$ Instituto de Biologia, Universidade Federal da Bahia, Salvador, Brasil \\ ${ }^{2}$ Programa de Pós-Graduação em Genética e Biodiversidade, Instituto de Biologia, Universidade Federal da \\ Bahia, Salvador, Brasil \\ ${ }^{3}$ Universidade Estadual de Feira de Santana, Departamento de Ciências Biológicas, Bahia, Brasil \\ ${ }^{4}$ Superintendência do IBAMA, Aracaju, Brasil \\ ${ }^{5}$ Universidade Federal da Bahia, Salvador, Brasil
}

\begin{abstract}
Resumo
A família Cactaceae possui entre 1500-1800 espécies agrupadas em 4 subfamílias. Embora a quantidade de estudos citogenéticos não cubra ainda parte significativa da família, alguns aspectos cromossômicos podem ser destacados. A família apresenta número básico $n=11$, com variações em ploidia em todas as quatro subfamílias, mas em um número limitado de gêneros. O fenômeno é mais comum em Opuntioideae, que apresenta reprodução vegetativa acentuada. Da mesma maneira, em outros grupos, muitos dos poliploides também reproduzem assexuadamente. Alguns táxons podem mostrar variação interespecífica, com diferentes ploidias. Os cariótipos são simétricos, denotando pouca alteração estrutural nos cromossomos que são pequenos, na maioria dos casos não excedendo $3 \mu \mathrm{m}$. Existem registros de híbridos interespecíficos e mesmo intergenéricos parcialmente férteis, o que denota uma razoável homologia dos cromossomos e, em alguns poliploides, foi detectado alto nível de formação de bivalentes.
\end{abstract}

Palavras-chave: Cariótipo, citogenética, poliploidia.

\begin{abstract}
The Cactaceae family has 1500-1800 species grouped into 4 subfamilies. Although the amount of cytogenetic studies have not yet cover a significant part of the family, some chromosomal aspects can be highlighted. Family presents basic number $n=11$, with variations in ploidy in all four subfamilies, but in a limited number of genus. The phenomenon is more common in Opuntioideae, which presents pronounced vegetative reproduction. Similarly, other groups, many of polyploid also reproduce asexually. Some taxa may show interspecific variation, with different levels of ploidy. The karyotypes are symmetrical, showing little structural change in chromosomes that are small, in most cases not exceeding 3 micrometers. There are records of interspecific hybrids and even partially fertile intergeneric which denotes a reasonable homology of chromosomes, and in some polyploid high level of bivalent formation was detected.
\end{abstract}

Keywords: Karyotype, cytogenetics, polyploidy.

* jose.geraldo.assis@terra.com.br

Recebido: 17/03/2014 Aceito: 17/03/2014 


\section{Introdução}

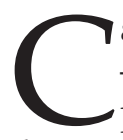

actaceae Juss. (Caryophyllales, Angiospermae - APG III, 2009) caracteriza-se por apresentar plantas extremamente adaptadas a condições áridas, apresentando caule e ramos suculentos e folhas muito reduzidas e/ou modificadas em espinhos, pela presença de aréolas e flores solitárias com ovário ínfero e receptacular (GIBSON e NOBEL, 1986; ANDERSON, 2001; NYFFELER, 2002).

A ampla diversidade da família Cactaceae está bem refletida no seu grande número de espécies. Estima-se entre 1500-1800 espécies distribuídas em 124 gêneros e quatro subfamílias (Maihuenoideae, Pereskeoideae, Opuntioideae e Cactoideae), com ocorrência natural quase exclusivamente nas Américas (exceto por Rhipsalis Gärtner, também encontrado na África e Ásia; BARTHLOTT, 1983), constituindo a segunda família mais numerosa das angiospermas neotropicais (TAYLOR, 2000; ANDERSON, 2001; BARTHLOTT e HUNT 1993; HUNT et al., 2006). Estudos filogenéticos têm demonstrado a monofilia das cactáceas (APPLEQUIST e WALLACE, 2001; CUÉNOUD et al., 2002; NYFFELER, 2002; HERNÁNDEZ-HERNÁNDEZ et al., 2011), no entanto, os estudos moleculares da família ainda são escassos, apesar de tratar-se de grupo com taxonomia complexa e com frequente convergência de caracteres morfológicos. Neste contexto, a citogenética de cactáceas tem contribuído na compreensão de importantes aspectos evolutivos e filogenéticos. Alguns estudos procuram também detectar alguma correlação entre morfologia e ploidia em espécies que apresentam morfotipos interespecíficos (POWELL e WEEDIN, 2001).

Apesar da sua ampla distribuição, as cactáceas constituem um dos grupos mais ameaçados de angiospermas, especialmente devido à expansão agrícola e ao extrativismo de espécimes para comercialização como plantas ornamentais (LONE et al., 2009).

\section{Poliploidia em Cactaceae}

Segundo Ortolani et al. (2007), um dos primeiros trabalhos com citogenética de cactáceas foi produzido por Stockwell, em 1935, quando analisou 45 espécies da família. Com os avanços de investigações em diversas espécies confirmou-se que a família apresenta número básico $n=11$ com poucas ocorrências de variações numéricas. Entretanto, a poliploidia teve um papel fundamental na evolução das cactáceas, sendo responsável por eventos de especiação (GIBSON e NOBEL, 1986). Na Tabela 1 são apresentados exemplos de gêneros, distribuídos em diferentes subfamílias e tribos em que a poliploidia já foi detectada.

Nota-se que a família é tipicamente diploide e a ocorrência de poliploidia é mais frequente em Opuntioideae, que apresenta série poliploide $(2 \mathrm{n}=22,44,66,88)$, por apresentar reprodução vegetativa como principal mecanismo reprodutivo, o que favorece o estabelecimento de poliploides na natureza (ROSS, 1981; NEGRON-ORTIZ, 2007). A poliploidia em cactáceas está correlacionada também com autofertilidade, embrionia adventícia, ramificação profusa, além da reprodução vegetativa (ROSS, 1981), e exemplos serão citados ao longo desta revisão.

Segundo Majure et al. (2012a) das 164 espécies de Opuntioideae para os quais contagens cromossômicas foram realizadas, $26,2 \%$ são diploides $13,4 \%$ são diploides e poliploides e $60,4 \%$ são poliploides. Em trabalhos que investigaram várias espécies, são poucas as que apresentaram registros exclusivamente diploides (PINKAVAet al., 1978; BANDYOPADHYAY \& SHARMA, 2000; PIMIENTA-BARRIOS \& MUÑOZ-URÍAS, 2001; POWELL \& WEEDIN, 2001; SEGURA et al., 2007). Em alguns tánons foram registrados citótipos poliploides e diploides: Opuntia aggeria Ralston \& Hilsenb., O. azurea Rose, O. davisii Engelm. \& J.M.Bigelow, O. leptocaulis DC.(PINKAVA et al., 1973; POWELL \& WEEDIN, 2001), O. polyacantha Haw.(KIESLING, 1998; PIMIENTA-BARRIOS \& MUÑOZ-URÍAS, 2001), O. robusta H.L.Wendl. ex Pfeiff. (PIMIENTA-BARRIOS \& MUÑOZ-URÍAS, 2001; SEGURA et al., 2007), O. schottii Engelm., O. stanlyi Engelm. (ORTOLANI et al., 2007), O. streptacantha Lem. (KIESLING, 1998; PIMIENTA-BARRIOS \& MUÑOZ-URÍAS, 2001; SEGURA et al., 2007).

A poliploidia também foi encontrada em outras Opuntioideae como no gênero Consolea Lem., endêmico das ilhas caribenhas, que contém nove espécies, entre as quais foram encontradas sete hexaploides e duas octoploides (NEGRON-ORTIZ, 2007), e em Cumulopuntia recurvata Gilmer \& H.P.Thomas, com $2 n=44$ (LAS PEÑAS et al., 2009).

Em espécies de Opuntioideae de ocorrência no Estado da Bahia, temos observado a ocorrência de espécies diploides como Opuntia palmadora Britton \& Rose, $O$. brasiliensis Haw. e Tacinga funalis Britton \& Rose, e poliploides como O. inamoena K.Schum., O. saxatilis(Ritter) Braun \& Pereira $(2 \mathrm{n}=4 \mathrm{x}=44)$ e O. werneri Eggli $(2 \mathrm{n}=$ $4 \mathrm{x}=44$ e $2 \mathrm{n}=6 \mathrm{x}=66)$. Castro (2008) também aponta o número $2 \mathrm{n}=22$ para O. palmadora, mas já encontramos indivíduos poliploides nesta espécie, proveniente do Município de Abaíra, na Bahia.

Dentre as espécies introduzidas, foi observada Opuntia cochenillifera (L.) Miller como diploide e $O$. dillenii Haw. com citótipos diploides e tetraploides. A espécie introduzida e cultivada como forrageira Opuntia ficus -indica (L.) Mill. apresentou $2 n=8 x=88$, constituindo um octoploide. Estudos anteriores relataram números gaméticos de 11 cromossomos (WEEDIN e POWELL, 1978), de 44 cromossomos (MOORE, 1973) e outra de 44 cromossomos que se refere a O. ficus-indica 'megacantha' (GOLDBLAT, 1984; PIMIENTA-BARRIOS \& MUÑOZ-URÍAS, 2001; SEGURA et al., 2007).É possível que o fato de esta espécie ser cultivada favoreça ocorrência de níveis tão variados de ploidia, que variam de 2x a 
Tabela 1. Gêneros de cactáceas estudados citogeneticamente indicando aqueles em que a poliploidia foi detectada em Cactáceas

\begin{tabular}{|c|c|c|}
\hline $\begin{array}{l}\text { Subfamília/tribo } \\
\text { ( }{ }^{\circ} \text { de gêneros) }\end{array}$ & $\begin{array}{l}\text { Gêneros em que não se verificou ocorrência de } \\
\text { poliploidia }\end{array}$ & $\begin{array}{l}\text { Gêneros em que se verificou ocorrência de } \\
\text { poliploidia }\end{array}$ \\
\hline $\begin{array}{l}\text { Maihuenioideae } \\
(1 \text { gênero })\end{array}$ & Maihuenia Phil. & \\
\hline $\begin{array}{l}\text { Pereskioideae } \\
\text { (1 gênero) }\end{array}$ & Pereskia Mill. & \\
\hline $\begin{array}{l}\text { Opuntioidade } \\
\text { (15 gêneros) }\end{array}$ & Nopalea Salm-Dick & $\begin{array}{l}\text { Austrocylindropuntia Backeb. } \\
\text { Consolea Lem. } \\
\text { Cylindropuntia (Engelm.) M.Knuth } \\
\text { Opuntia Mill. } \\
\text { Pereskiopsis Vaupel in Engler e Prantl } \\
\text { Pterocactus K. Schum. } \\
\text { Quiabentia Britton \& Rose } \\
\text { Tacinga Britton \& Rose }\end{array}$ \\
\hline $\begin{array}{l}\text { Cactoideae/Notocacteae } \\
\text { (10 gêneros) }\end{array}$ & $\begin{array}{l}\text { Eriosyce Phil. (= Pyrrhocactus A.Berger) } \\
\text { Frailea Britton e Rose } \\
\text { Neoporteria Britton \& Rose }\end{array}$ & $\begin{array}{l}\text { Blossfeldia Werderm. } \\
\text { Sulcorebutia Backeb. } \\
\text { Notocactus (K.Schum. Frič) }\end{array}$ \\
\hline $\begin{array}{l}\text { Cactoideae/Trichocereeae } \\
\text { (26 gêneros) }\end{array}$ & $\begin{array}{l}\text { Discocactus Pfeiff. } \\
\text { Espostoopsis Buxb. } \\
\text { Facheiroa Britton \& Rose } \\
\text { Harrisia Britton } \\
\text { Leocereus Britton \& Rose } \\
\text { Uebelmannia Buining }\end{array}$ & $\begin{array}{l}\text { Cleistocactus Lem. } \\
\text { Echinopsis Zucc. } \\
\text { Espostoa Britton \& Rose } \\
\text { Gymnocalycium Pfeiff. ex Mitler } \\
\text { Haageocereus Backeb. } \\
\text { Lobivia Britton \& Rose } \\
\text { Rebutia K.Schum. } \\
\text { Weberbauerocereus Backeb. }\end{array}$ \\
\hline $\begin{array}{l}\text { Cactoideae/Cereeae } \\
\text { ( } 7 \text { gêneros) }\end{array}$ & $\begin{array}{l}\text { Arrojadoa Britton \& Rose } \\
\text { Brasilicereus Backeb. } \\
\text { Cipocereus F.Ritter } \\
\text { Coleocephalocereus Backeb. } \\
\text { Micranthocereus Backeb. } \\
\text { Pilosocereus Byles \& G.D.Rowley } \\
\text { Praecereus Buxb. } \\
\text { Stephanocereus Berger. }\end{array}$ & $\begin{array}{l}\text { Cereus Mill. } \\
\text { Melocactus Link \& Otto. }\end{array}$ \\
\hline $\begin{array}{l}\text { Cactoideae/Cacteae } \\
\text { (18 gêneros) }\end{array}$ & $\begin{array}{l}\text { Ancistrocactus Britton \& Rose } \\
\text { Astrophytum Lem. } \\
\text { Coryphanta (Engelm.) Lem. } \\
\text { Ferocactus Britt. \& Rose } \\
\text { Neolloydia Britton \& Rose } \\
\text { Pediocactus Britton \& Rose } \\
\text { Pelecyphora Ehrenb. } \\
\text { Strombocactus Britton \& Rose } \\
\text { Thelocactus (Schum) Britton \& Rose } \\
\text { Sclerocactus Britton \& Rose }\end{array}$ & Mammillaria Haw. \\
\hline $\begin{array}{l}\text { Cactoideae/Hylocereeae } \\
\text { (6 gêneros) }\end{array}$ & $\begin{array}{l}\text { Cryptocereus Alexand. } \\
\text { Disocactus Lindl. } \\
\text { Epiphyllum Haw. } \\
\text { Nopalxochia Britton \& Rose } \\
\text { Nyctocereus (Berger) Britton \& Rose } \\
\text { Weberocereus Britton \& Rose }\end{array}$ & $\begin{array}{l}\text { Hylocereus Britton \& Rose } \\
\text { Mediocactus Britton \& Rose } \\
\text { Selenicereus (Berger) Britton \& Rose }\end{array}$ \\
\hline $\begin{array}{l}\text { Cactoideae/Rhipsalideae } \\
\text { (4 gêneros) }\end{array}$ & $\begin{array}{l}\text { Hatiora Britton et Rose } \\
\text { Lepismium Pfeiff }\end{array}$ & $\begin{array}{l}\text { Rhipsalis Gaertn. } \\
\text { Schlumbergera Lem. }\end{array}$ \\
\hline $\begin{array}{l}\text { Cactoideae/Echinocereae } \\
\text { (4 gêneros) }\end{array}$ & & Echinocereus \\
\hline $\begin{array}{l}\text { Cactoideae/Pachycereae } \\
\text { (19 gêneros) }\end{array}$ & Corryocactus Britton \& Rose & $\begin{array}{l}\text { Stenocereus (Berger) Riccob. } \\
\text { Cephalocereus Pfeiff. } \\
\text { Myrtillocactus Console } \\
\text { Echinocereus Engelm }\end{array}$ \\
\hline
\end{tabular}


8x (MAJURE et al., 2012a). Além disso, características peculiares cariotípicas, como tamanho cromossômico maior que o comumente encontrado no gênero, foram encontradas em um citótipo pentaploide da espécie por Bandyopadhyay \& Sharma (2000) e apontam uma posição separada no complexo Opuntia ficus-indica.Griffith (2004), baseado em estudo de sequências de DNA de ITS, defendeu a hipótese de que o conceito taxonômico desta espécie cultivada inclui clones derivados de múltiplas linhagens parentais, tratando-se de um grupo polifilético, no qual eventos de poliploidia e hibridação teriam ocorrido.

A hibridação entre outros opuntioides foi relatada entre os poliploides Consolea rubescens Salm-Dyck ex DC. e O. dillenii, C. nashii Britton e O. dillenii e C. rubescens e O. dillenii (NEGRÓN-ORTIZ, 2007).

Além da ocorrência em Opuntioideae, a poliploidia foi identificada, também, em gêneros de Cactoideae, incluindo Gymnocalycium Pffeif., Mammillaria Haw., Rebutia K.Schum. e Rhipsalis Gaertn. (GIBSON e NOBEL, 1986). À época eram citados de 15 gêneros e outros registros foram obtidos posteriormente, elevando ainda mais este número. Nas tribos Cereeae e Trichocereeae, que formam junto com Browningeae um clado irmão do grupo Notocacteae (NYFFELER, 2002), a ocorrência de poliploides é mais destacada, talvez, pela maior representatividade destes, e, consequentemente, de maiores esforços em pesquisa. Este clado, denominado BCT, compreende 20 gêneros e 400 espécies da maioria dos cactos colunares e globulares da América do Sul (NYFFELER, 2002). Tribos menos representativas de Cactoideae, como Leptocereae e Browningeae, não apresentaram, até o momento, ocorrência de poliploides, ou sequer foram incluídos em estudos citogenéticos. Leptocereae constitui, segundo Gibson e Nobel (1986), o grupo de Cactoideae que apresenta a maioria dos caracteres primitivos.

Na tribo Cacteae, o gênero com número destacado de poliploides é Mammillaria, incluindo as espécies tetraploides M. compressa DC., M. multiceps Salm-Dyck, $M$. parkinsonia Ehremberg, e M. prolifera (Mill.) Haw. (ROSS, 1981; MOHANTY et al., 1996). Esta última apresenta reprodução assexuada (ROSS, 1981), incomum no gênero, confirmando a tendência de surgimento de poliploidia em plantas com este tipo de reprodução. Por outro lado, gêneros como Ferocactus Britton \& Rose, que tiveram inúmeras espécies estudadas, mostraram uniformidade diploide (PINKAVA et al., 1973, COTA et al., 1996).

Dentro da tribo Hylocereae, o gênero Selenicereus (A.Berger) Britton \& Rose apresenta espécies tetraploides como S. megalanthus (K.Schum. ex Vaupel) Moran (LICHTENZVEIG et al., 2000) e Selenicereus setaceus Salm-Dyck ex De Candolle (= S. rizzinii Scheinvar) (dados dos autores). Por outro lado, Hylocereus undatus (Haw.) Britton\& Rose e H. polyrhizus (F.A.C.Weber) Britton \& Rose, que são intercruzáveis com $S$. megalanthus, são diploides (LICHTENZVEIG et al., 2000). Também é di- ploide a espécie Hylocereus monacanthus (Lem.) Britton\& Rose (CISNEROS et al., 2011).

O estado diploide dentro da tribo Hylocereae tem sido encontrado em estudos de outros gêneros como Epiphyllum (MOORE, 1973; GOLDBLATT, 1981), Weberocereus (LICHTENZVEIG et al., 2000) e Mediocactus, embora este último apresente M. coccineus (Salm-Dyck) Br. \& R., descrita como tetraploide. Este gênero, entretanto, tem sido considerado sinonímia de Selenicereus (LICHTENZVEIG et al., 2000).

Na tribo Echinocereae foram encontrados poliploides em variedades de Echinocereus enneacanthus Engelm. e Echinocereus pectinatus Engelm., e Echinocereus triglochidiatus Engelm. (PINKAVA et al., 1973; WEEDIN e POWELL (1978).

Em Rhipsalideae destaca-se o gênero de espécies epífitas Rhipsalis, com espécies diploides, tetraploides e octoploides. A espécie R. baccifera (Sol.) Stearn, com seis subspécies, é o único cacto exibindo faixa de disjunção transatlântica, ocorrendo na África Central e em Madagascar (provável centro secundário com formas poliploides), Ilhas Seychelles, Ilha Maurício (República da Maurícia) e Sri Lanka (COTA-SÁNCHEZ e BOMFIM -PATRÍCIO, 2010).

$\mathrm{Na}$ tribo Notocacteae encontra-se a hexaploide $(2 \mathrm{n}$ = 66) Blossfeldia liliputana Werderm. (ROSS, 1981), uma espécie de reprodução assexuada. Segundo HERNÁNDEZ-HERNÁNDEZ et al. (2011) Blossfeldia Werderm. é um gênero monotípico, distribuído entre Argentina e Bolívia. São plantas pequenas com características morfológicas e ecológicas raramente encontradas em outros cactos. Embora esteja incluído em Notocacteae (Cactoideae), estudos moleculares baseados em dados de DNA cloroplasmático demonstraram que Blossfeldia constitui uma linhagem distinta que constitui o grupo irmão de Cactoideae e Rhipsalidodeae, e que deveria ser reconhecida como uma nova subfamília, Blossfeldioideae, (CROIZER, 2004). A divergência de Blossfeldia em Cactoideae também havia sido relatada por Nyffeler (2002). Outros autores, entretanto, defendem sua manutenção em Cactoideae, como sugerido pelas evidências morfológicas, uma vez que uma possível origem híbrida e evolução reticulada de Blossfeldia ainda não foram investigadas (GORELICK, 2004).

A tribo Cereeae apresenta poliploides em diversas espécies do gênero Melocactus, como M. matanzanus Leon, de ocorrência em Cuba, e em diversas espécies do leste do Brasil, como relatado por Assis et al. (2003). São poliploides: Melocactus bahiensis (Britton \& Rose) Luetzelb. subsp. amethystinus Buining \& Brederoo N.P. Taylor, M. conoideus Buining \& Brederoo, M. glaucescens Buining \& Brederoo, Melocactus oreas Miq., M. oreas Miq. subsp. cremnophilus (Buining \& Brederoo) P.J.Braun, M. paucispinus G.Heimen \& R.Paul, M. salvadorensis Werderm., M. ernestii Vaupel e M. zehntneri (Britton \& Rose) Luetzelb., as duas últimas também com citótipos diploides. Também nesta tribo encontrou-se um poliploi- 
de no gênero Cereus Mill. entre seis espécies estudadas (PINKAVA et al., 1973).

Além de casos já citados em membros de Opuntioideae, a ocorrência de variação intraespecífica de ploidia também foi detectada em Cactoidae. Em Melocactus ernestii Vaupel e M. zehntneri (Britton \& Rose) Luetzelb. foram encontrados citótipos diploides e tetraploides (ASSIS et al., 2003), e é possível haver também variação em M. concinnus Buining \& Brederoo e $M$. albicephalus Buining \& Brederoo, uma vez que Assis et al. (2003) as descreveram como diplóides e Das et al. (1998a) como tetraploides.

Em estudos desenvolvidos no nosso laboratório, não encontramos poliploidia nas espécies brasileiras: Arrojadoa rhodantha Britton \& Rose, A. penicillata Britton \& Rose, Cereus jamacaru DC., Coleocephalocereus aureus F.Ritter var. brevicylindricus (Buining) P.J.Braun, C. goebelianus (Vaupel) Buining, Micranthocereus flaviflorus Buining \& Brederoo, M. purpureus (Gürke) F.Ritter, Pilosocereus gounelli (F.A.C.Weber ex K.Schum.) Byles \& G.D.Rowley, P.magnificus (Buining \& Brederoo) F.Ritter, P. multicostatus F.Ritter, P. pentaedrophorus (Labour.) Byles \& G.D.Rowley, e P. quadricostatus F.Ritter.

A tribo Trichocereeae apresenta vários registros de gêneros poliploides. Em Gymnocalycium, G. bruchii (Speg.) Hoss, apresenta 2n $=44$ (ROSS, 1981). Mihalte et al (2011) analisaram 20 espécies pertencentes a quatro gêneros de Cactaceae (Rebutia K.Schum., Aylostera Speg., Mediolobivia Backeb. e Sulcorebutia Backeb.) e encontraram duas espécies tetraploides (Sulcorebutia crispata Rausch e Rebutia kupperiana Boed. var. spiniflora F.Ritter, $2 \mathrm{n}=$ $4 \mathrm{x}=44)$ e cinco espécies triploides (R. pseudodeminuta Backeb. var. schumanniana Backeb., R. senilis Backeb. var. lilacinorosea Backeb., R. calliantha Bewer., R. donaldiana A.B.Lau \& G.D.Rowley e Mediolobivia diersiana Rausch, $2 n=3 x=33$ ). As outras treze espécies foram diploides com $2 n=2 x=22$. Ross (1981) citou como poliploides as espécies Rebutia kupperiana Boed., R. spegazziana Backeb., além de Rebutia sp. cultivar 'nivea'. Estas três espécies têm reprodução assexuada.

Arakaki et al. (2007) analisaram 40 táxons, dos quais 35 de oito gêneros de Trichocereae, sendo 17 do gênero Haageocereus Backeb. Apresentaram contagens poliploides Espostoa Britton \& Rose, Cleistocactus Lem., Haageocereus, e Weberbauerocereus Backeb., tendo sido detectados um triploide, nove tetraploides, um hexaploide, e três octoploides.

Do gênero Harrisia, nós determinamos número diploide para H. adscendens (Gürke) Britton \& Rose. A inclusão deste gênero em Trichocereae, entretanto, tem sido questionada (APPLEQUIST e WALLACE, 2002).

Schlumberger e Renner (2012) analisaram o número cromossômico de diversas espécies (e subespécies ou variedades) de Echinopsis Zucc.,além de outras 11 espécies de grupos pertencentes a oito gêneros de Trichocereeae e, ainda, relataram casos de tetraploidia e de variação intraespecífica entre diploidia e tetraploidia.
Os tetraploides encontrados foram: Oreocereus celsianus (Salm-Dyck) A.Berger ex Riccob., O. pseudofossulatus D.R.Hunt,Echinopsis candicans (Salm-Dyck) D.R.Hunt, E. jajoiana Hort. ex Blossf., E. thelegonoides (Speg.) H. Friedrich \& G.D.Rowley, Rebutia atrovirens (Backeb.) Pilbeam, R. haagei Frič \& Schelle, R. pygmaea Britton \& Rose, Weberbauerocereus weberbaueri (K.Schum. ex Vaupel) Backeb., e Espostoa sp.. A ocorrência de poliploidia em Echinopsis pode estar relacionada com a reprodução clonal apresentada neste gênero, onde se relata a ocorrência de baixa reprodução sexuada e poucos frutos e sementes produzidos (NEGRÓN-ORTIZ, 1998; ORTEGA-BAES e GOROSTIAGUE, 2013).

Variação intraespecífica foi detectada em Echinopsis tiegeliana (Wessner) D.R.Hunt, em Haageocereus pseudomelanostele (Werderm. \& Backeb.) Backeb. (com 2n=22/33/44), e em Rebutia heliosa Rausch e R. muscula F.Ritter \& Thiele, espécies anteriormente designadas como Mediolobivia Backeb. (SCHLUMBERGER e RENNER, 2012). Outras espécies da tribo Trichocereeae com variação interespecífica são Espostoa lanata (Kunth) Britton \& Rose, com $2 \mathrm{n}=22$, 44, e 66, e em Weberbauerocereus rauhii Backeb., com $2 \mathrm{n}=44$ e 88 (ARAKAKI et al., 2007).

Nas duas subfamílias mais basais, Pereskioideae e Maihuenioideae, os relatos apontam para um estado diploide. Segundo Taylor (2000), a subfamília Pereskioideae possui apenas um único gênero: Pereskia Mill., constituído por 17 espécies, sendo 5 nativas do leste do Brasil. São comumente citados como os cactos mais primitivos por apresentarem numerosos caracteres plesiomórficos (caules não suculentos, folhas bem desenvolvidas e persistentes, inflorescência cimosa). Algumas espécies de Pereskia apresentam ovário semi-ínfero, ou até mesmo súpero, com placentação basal, enquanto as demais cactáceas possuem ovário ínfero com placentação parietal (JUDD et al., 2007). O número cromossômico de praticamente todas as espécies de Pereskia já foi elucidado (PINKAVA et al., 1973; GOLDBLATT, 1984 e 1990). Nossos estudos em P. bahiensis Gürke e P. grandifolia Haw. confirmaram contagens anteriores de $2 n=$ 22 para o gênero. Da mesma maneira, Las Peñas et al. (2009) encontraram este número para P. aculeata Mill. e Ross (1981) em P. corrugata Cutak. Em Maihuenioidae, entretanto há registro de uma espécie (Maihuenia patagonica Britton \& Rose) com citótipos diploide e tetraploide (LAS PEÑAS et al., 2009).

Os níveis de ploidia em cactáceas, segundo Baker et al. (2009), estão geralmente correlacionados com a distribuição geográfica e morfológica das espécies e são cruciais na evolução e sistemática da família. O aspecto mais importante da poliploidia, de acordo com estes autores, é que cria uma barreira genética (isolamento reprodutivo sexual) que geralmente leva à especiação. Estes autores analisaram os padrões de distribuição espacial de citótipos de seis espécies de Cylindropuntia (Engelm.) F.M. Knuth. Em geral, eles observaram os poliploides ocupando áreas distintas dos diploides, 
muitas vezes em áreas marginais à área de distribuição da espécie. As populações sulamericanas de Rhipsalis baccifera no Brasil, Bolívia e Paraguai são principalmente diploides, enquanto as do México, Costa Rica e Caribe são tetraploides. As populações da África Central e Madagascar são tetra e octoploides (COTA-SÁNCHEZ \& BOMFIM-PATRÍCIO, 2010).

Os poliploides do gênero Haageocereus, identificados por Arakaki et al. (2007), ocorrem em ambientes extremamente mais severos em comparação com a maioria das espécies diploides do gênero. Segundo esses autores, diversas populações de Haageocereus acranthus (Vaupel) Backeb.e H. tenuis F.Ritter, vivem em condições de extrema aridez, nas quais não são encontrados diploides.

Com relação à morfologia das plantas, Majure et al. (2012a) detectaram diferenciação morfológica entre citótipos diploides e poliploides de Opuntia e indicam que tais distinções podem ser úteis no diagnóstico de possíveis espécies crípticas.Arakaki et al. (2007) notaram que a maioria dos poliploides de Weberbauerocereus mostram cor do caule verde azulado escuro e número de ramificações reduzido em relação aos diploides. Cota-Sánchez e Bomfim-Patrício (2010) mostraram um aumento no tamanho das sementes relacionado ao nível de poliploidização em Rhipsalis, tendo expressão máxima nas populações poliploides da África. Observaram correlação, também, com o tamanho do caule e do fruto e número de estômatos. Não houve, entretanto, correlação com o tamanho das células dos estômatos.

Segundo Pierce (2004), em plantas a poliploidia é um importante meio de permitir a evolução de novas espécies e, em geral, a poliploidia propicia um aumento no tamanho das células ocasionando que a maior parte dos poliploides seja maior que os diploides que lhes originaram. Dessa forma, devido ao surgimento de novas características, a formação de poliploides geralmente acarreta a dispersão destes indivíduos da sua área original, propiciando a exploração de novos habitats nos quais não haverá competição com os diploides que os originaram.

Assim, os poliploides apresentam, em sua maior parte, uma área maior de distribuição do que os diploides aparentados, haja vista que o local em que estão presentes os diploides seja um centro de diversificação primária e as espécies que se irradiam a partir desse local (os poliploides) possuem mais chance de adaptação em novos habitats que apresentem características distintas do centro de origem (ARAKAKI et al., 2007).

Outro exemplo da existência de relação entre a variação citológica e a distribuição geográfica em Cactaceae vem do gênero Opuntia. A rota evolutiva da espécie Opuntia polyacantha Haw. apresenta uma linhagem diploide no norte do México e derivados tetraploide e hexaploide nos Estados Unidos e Canadá, respectivamente (PINKAVA, 2002). Por outro lado, Segura et al. (2007) não encontraram nenhuma correlação em estudo envolvendo 23 espécies do gênero. Majure et al. 2012c descreveram populações diploides, triploides e tetraploides de O. pusilla Haw. numa faixa restrita do sudeste dos Estados Unidos, sendo que os diploides estavam situados na parte interior da distribuição e quase todos os poliploides na linha costeira.

\section{Aneuploidia em Cactaceae}

Segundo Baker et al. (2009), a aneuploidia parece ter pouca importância na evolução dos cactos, considerando que quase todas as determinações cromossômicas têm sido euploides e apenas 16 táxons foram relatados como aneuploides, embora destas existam registros para 14 delas como euploides. Entretanto, a maioria destes relatos é dos anos 1930s, quando as técnicas para determinação cromossômica não tinham tanta confiabilidade como nos dias atuais. Essas contagens aneuploides não foram repetidas. Um destes aneuploides relatados foi Deamia (= Selenicereus testudo (Karw.) Britton\& Rose) com n =12, determinado por Batttachoryya em 1970 (ROSS, 1981). Pinkava et al. (1973) registraram um indivíduo trissômico no complexo O. phaeacantha Engelm.

\section{Estudos cariotípicos}

A despeito da pouca variação numérica, os detalhamentos cariotípicos têm sido úteis em alguns casos na compreensão de relações filogenéticas. Os cromossomos de cactáceas são, em geral, pequenos e em toda a família mostram predominância do tipo metacêntrico e os cariótipos são simétricos.

Em vinte espécies dos gêneros Rebutia, Sulcorebutia, Aylostera e Mediolobivia, Mihalte et al. (2011) verificaram que os cromossomos não excediam $2 \mu \mathrm{m}$. Las Peñas et al. (2009), analisando espécies das 4 subfamílias, relataram que o comprimento médio dos cromossomos era de 2,3 $\mu \mathrm{m}$, fato corroborado também por Ortolani et al., 2007. Em três espécies de Opuntia Palomino e Heras (2001) encontraram os menores cromossomos variando de 1,07 a 1,55 $\mu \mathrm{m}$ e os maiores de 2,5 a 3,57 $\mu \mathrm{m}$. Estudos nossos com diferentes espécies nativas, a amplitude encontrada foi de menos de $1 \mu \mathrm{m}$ a 2,49 $\mu \mathrm{m}$. Castro (2008), também estudando diversas espécies brasileiras, verificou amplitude de 0,9 a 5,0 $\mu \mathrm{m}$ sendo os maiores (acima de $3 \mu \mathrm{m}$ ) encontrados principalmente na subfamília Pereskioideae. Las Peñas et al. (2008) encontraram em sete espécies de Pyrrhocactus A.Berger tamanhos cromossômicos médios variando de 2,2 a 3,10 $\mu \mathrm{m}$. Briones et al. (2004) observaram em três espécies de Mammillaria variação entre 1,79 a 3,21 $\mu \mathrm{m}$, de modo geral, maiores do que determinado anteriormente para M. sanangelensis Sánches-Mej $(0,80$ $1,70 \mu \mathrm{m})$, que podem ser considerados os mais curtos do gênero (PALOMINO et al., 1999 apud BRIONES et al., 2004). Tamanhos cromossômicos maiores foram descritos por Arakaki et al. (2007), que encontraram variações entre 
3 a 5 m em 40 espécies de 13 gêneros de Cactaceae, a maioria pertencente a tribo Trichocereae, Cactoideae.

A incongruência entre estes dados de tamanho cromossômico deve estar relacionada aos diferentes métodos e recursos laboratoriais de mensuração, mas é unânime o entendimento de que se tratam de cromossomos pequenos. Na Tabela 2 são mostradas médias ou faixas de variação do tamanho dos cromossomos individuais e do tamanho do comprimento do genoma haploide em diferentes gêneros estudados.

Possivelmente é devido a esse reduzido comprimento que, conforme Ortolani et al. (2007), a produção bibliográfica em citogenética de cactáceas é escassa. Isso ocorre, de acordo com Guerra (1988), devido às grandes dificuldades de se detalhar a estrutura cromossômica, restando apenas a possibilidade de se realizar a contagem de cromossomos.

A predominância de cromossomos metacêntricos foi evidenciada em todas as subfamílias de Cactaceae. Na maioria dos estudos cariotípicos, quando não são todos os cromossomos metacêntricos, encontra-se apenas um ou dois submetacêntricos em todo o conjunto (BRIONES et al., 2004; DEL ANGEL et al., 2006; LAS PEÑAS et al., 2009), com exceção Schlumbergera, no qual foram encontrados 5 cromossomos submetacêntricos em duas espécies (ORTOLANI et al., 2007).

Estudos cariotípicos foram conduzidos em três espécies octoploides de Opuntia (Opuntia cochinera Griffths, O. hyptiacantha F.A.C.Weber e O. strepthacantha Lem.) do Vale do México, apresentando, todas elas, a totalidade dos cromossomos do tipo metacêntrico, mas com variações no tamanho dos cromossomos, sendo que o compri- mento do conjunto genômico variou de 152,49 a 210,99 $\mu \mathrm{m}$ (PALOMINO e HERAS, 2001). Nestes poliploides, foram observados seis cromossomos com satélites em O. hyptiacantha e 20 em O. strepthacantha, indicando que ambas têm origem alooctoploide.

No entanto, a presença de satélites nos cromossomos de cactáceas é raramente registrada e, nestes casos, para poucos cromossomos. Las Peñas et al. (2008) encontraram dois pares em sete espécies de Pyrrhocactus, Palomino et al. (1999) encontraram apenas um par em Mammillaria sanangelensis e Las Peñas et al. (2009) registraram um cromossomo satelitizado nas espécies diploides estudadas de Cumulopuntia e no tetraplóide de $C$. recurvata, enquanto que nos demais tetraploides foram encontrados quatro. Bandyopadhyay \& Sharma (2000) encontraram 4 ou 6 cromossomos com satélites em 8 táxons de Opuntia, incluindo diploides e tetraploides. Em quatro espécies de Astrophytum Lem. o número de constrições secundárias variou entre 2 e 6 (DAS et al., 2000). Briones et al. (2004) detectaram um par de cromossomos satelitizado em Mammillaria supertexta Mart. e em M. haageana Pfeiff., enquanto que em $M$. crucigera Mart. foram encontrados dois pares. Das et al. (1998b) encontraram em 12 espécies deste gênero de dois a quatro cromossomos com constrição secundária. Castro (2008) relatou a presença de um a dois satélites em Arrojadoa Mattf., Nopalea Salm-Dyck e Pereskia, e dois pares de cromossomos com satélites em Pilosocereus e Melocactus. Em nosso laboratório detectamos pares de cromossomos com satélite em Pereskia bahiensis Gürke, P. grandiflora Haw., Harrisia adscendens (Gürke) Britton \& Rose, Discocactus placentiformis K.Schum. e D. zehnt-

Tabela 2. Variação do tamanho dos cromossomos individuais e do tamanho do comprimento do genoma haploide em espécies de cactáceas.

\begin{tabular}{|l|c|c|c|l|}
\hline Gênero & $\begin{array}{l}\text { No de táxons } \\
\text { estudados }\end{array}$ & $\begin{array}{l}\text { Tamanho dos } \\
\text { cromossomos } \\
(\mu \mathrm{m})\end{array}$ & $\begin{array}{l}\text { Variação na média } \\
\text { do comprimento ha- } \\
\text { ploide do genoma } \\
(\mu \mathrm{m})\end{array}$ & \multicolumn{1}{|c|}{ Referência } \\
\hline Mammillaria & 3 & 1,79 a 3,21 & 23.06 a 26.84 & Briones et al., 2004 \\
\hline Mammillaria & 4 & 1,56 a 3,73 & 22,98 a 30,37 & Del Angel et al., 2006 \\
\hline Pyrrhocactus & 7 & 1,3 a 3,6 & 22,0 a 35,3 & Las Peñas et al., 2008 \\
\hline Melocactus & 14 & ca. 2 & $40,0(2 \mathrm{n}=22)$ & Assis et al., 2003 \\
\hline Melocactus & 6 & $3,28(2 \mathrm{n}=22)$ & $72,26(2 \mathrm{n}=22)$ & Das et al., 1998 \\
& & $3,18(2 \mathrm{n}=44)$ & $160,05(2 \mathrm{n}=44)$ & $\begin{array}{l}\text { Bandyopadhyay } \\
\text { Sharma, 2000 }\end{array}$ \\
\hline Opuntia & 8 & 0,97 a 3,46 & & \\
\hline
\end{tabular}


neri subsp. boomianus(Buining \& Brederoo) N.P.Taylor \& Zappi.

Para análise comparativa de cariótipos de cactáceas, Das et al. (1998b, 2000) e Das e Mohanty (2006) utilizaram classificação dos cromossomos propondo-se quatro tipos. No tipo A os cromossomos apresentam tamanho médio e duas constrições; no tipo B, cromossomos possuem tamanho médio a pequeno, com uma constrição na posição sub-terminal e o satélite está localizado no braço longo do cromossomo; no tipo $\mathrm{C}$ os cromossomos têm tamanho de médio a pequeno, com constrições mediana; e, por fim, no tipo D os cromossomos são de tamanho médio a pequeno com constrições sub-medianas. Este sistema foi útil para diferenciar cariótipos de sete espécies de Echinopsis que apresentaram fórmulas distintas, com exceção de E. multiplex (Pfeiff.) Zucc. ex Pfeiff \& Otto e E. tubiflora Zucc.ex A.Dietr. Estas espécies, no entanto, apresentaram diferenças significativas no conteúdo de DNA nuclear, provavelmente relacionadas a diferenças no conteúdo de eucromatina e heterocromatina (DAS e MOHANTY, 2006).

A simetria dos cariótipos de cactáceas foi apontada por Las Peñas et al. (2009) para membros das quatro subfamílias, aspecto registrado também em quase todos os trabalhos que observaram este aspecto (Castro, 2008). Las Peñas et al. (2008) detectaram um aumento de assimetria em espécies de Pyrrhocactus associado à diminuição do tamanho do cariótipo. Os índices de assimetria em espécies de cactáceas encontram-se em geral entre 40 e $46,55 \%$, como observado para espécies de Mammillaria (BRIONES et al., 2004 e DEL ANGELet al., 2006). Em Opuntia, o índice variou de 33 a 51\% (BANDYOPADHYAY e SHARMA, 2000).

Alguns trabalhos relataram a ocorrência de ploidia utilizando-se da técnica de citofotometria de fluxo. São exemplos os estudos de Mohanty et al. (1996), Mohanty et al. (1997) e Das et al. (1998b), que estudaram diversas espécies de Mammillaria (com variação de 9,1 pg em $M$. klissingiana Boed. a 26,46 pg em M. plumosa F.A.C.Weber), Das et al. (2000), que estudaram quatro espécies de Astrophytum Lem. (que variaram de 9,23 pg a 10,12 pg), Das et al. (1998a e 1998b), que investigaram seis espécies de Melocactus e Mammillaria, respectivamente, e de Das \& Mohanty (2006), que investigaram sete espécies de Echinopsis. Neste último a variação foi de 7,353 pg a 10,352 pg. Segura et al. (2007) utilizaram a técnica no estudo de 23 espécies de Opuntia, tendo encontrado quatro diferentes níveis de ploidia. As quantidades de valor $2 \mathrm{C}$ de DNA variaram de 4,17 a 6,53 pg. Negron-Ortiz (2007), analisando também opuntióides poliploides, encontrou variação de 4,88 a 9,50 pg. Nestes trabalhos, as variações interespecíficas no conteúdo de DNA podem ser atribuídas a variações nas sequências de DNA repetitivo do genoma, indicando pequenas deleções ou adições do material nuclear durante a evolução.

O conteúdo estimado por Palomino et al. (1999) para Mammillaria sanangelensis foi inferior, sendo de apenas 3,2 pg. Segundo estes autores, estes valores assemelhavam-se aos encontrados por Barlow e Nevin (1976) para nove espécies de Luzula, que variaram de 3,1 a 4,1 pg, e ainda a Pereskia angustifolia (2,05 pg). Considerando a faixa do tamanho do genoma apresentado por angiospermas por Barlow e Nevin (1976) variando de 0,2 to 127,4 pg. Palomino et al. (1999) consideraram que, embora haja desacordos nas estimativas de valor 2C em diferentes trabalhos de cactáceas, as espécies da família apresentam pequeno genoma.

São raros os estudos cariotípicos envolvendo bandeamentos. Ortolani et al. (2007) utilizaram banda C para discriminar cariótipos de quatro variedades de Schlumbergera truncata (Haworth) Moran e da espécie híbrida Schlumbergera $\mathrm{x}$ buckleyi (T. Moore) Tjaden. Detectaram ausência total de bandas em uma das variedades de $S$. truncata. Em relação aos demais aspectos cariotípicos, pouca divergência foi detectada entre as duas espécies e/ou variedades.

Las Peñas et al. $(2008,2009)$ realizaram trabalhos pioneiros utilizando bandeamento CMA/DAPI e encontraram bandas CMA+/DAPI- associadas com bandas regiões organizadoras de nucléolo no par número 1 em todas as espécies examinadas nos dois trabalhos (sete espécies de Pyrrhocactus, Cumulopuntia recurvata, Acanthocalycium spiniflorum(K. Schum) Backeb. e Maihuenia patagonica) evidenciando homologia. Heterocromatina pericentromérica foi encontrada em $C$. recurvata, $A$. spiniflorum, e no citótipo tetraploide de M. patagonica. A localização de sítios 18S-26S rDNA coincidiram com bandas CMA+/DAPI- mostrando-se altamente conservado (LAS PEÑAS et al., 2008).

\section{Hibridização e estudos meióticos}

Para alguns autores, a alta similaridade na morfologia e no número de cromossomos explica, em parte, a facilidade com que os cactos produzem híbridos interespecíficos e até intergenéricos férteis (GIBSON e NOBEL, 1986; ARAKAKI et al., 2007). Outros fatores que podem favorecer o sucesso na natureza, ou mesmo em híbridos artificiais, é autofertilidade e a frequente reprodução assexuada (ROSS, 1981; ARAKAKI et al., 2007).

Em Opuntioidae, a evolução reticulada tem sido apontada como um mecanismo importante de evolução. Majure et al. (2012b) referem-se à 24 táxons híbridos dos quais 20 são alopoliploides.

Os relatos de hibridização têm motivado estudos meióticos de híbridos a fim de mensurar o nível de fertilidade. Por exemplo, Pinkavaet al. (1973) confirmaram a esterilidade em um híbrido triploide entre duas espécies de Cereus, que também apresentaram grãos de pólen inviáveis e formação de trivalentes e segregação irregular de cromossomos na meiose. Um caso bem estudado envolve uma hibridização intergenérica de espécies com diferentes níveis de ploidia Selenicereus vs Hylocereus. 
As espécies Hylocereus undatus e H. polyrhizus, são diploides enquanto Selenicereus megalanthus é tetraploide e nos cruzamentos interespecíficos a formação de frutos variou de 90 a $100 \%$, com alta produção de sementes e germinação de pólen in vivo (LICHTENZVEIG et al., 2000). Os autores mostraram que nos diploides Hylocerus undatus e H. polyrhizus há formação de pólen. Selenicereus megalanthus tem sido identificado mais recentemente como Hylocereus megalanthus (CISNEROS et al., 2011), de acordo com as evidências de intercruzabilidade que apresenta com as espécies de Hylocereus.

Os trabalhos que se dedicaram a estudos meióticos, seja para determinação do número cromossômico ou para avaliar a regularidade meiótica, permitiram avaliar melhor o nível de homologia cromossômica dentro da família. Lichtenzveig et al. (2000) verificaram que em dois clones tetraploides de Selenicereus megalanthus há formação de um número variável de univalentes (0-4) e multivalentes (0-6). Nos diploides Hylocereus undatus e $H$. polyrhyzus ocorre a formação de bivalentes e segregação regular na primeira divisão meiótica. Pode-se observar que a porcentagem de cruzamento bem sucedidos nos cruzamentos intergenéricos entre Selenicereus megalanthus e H. undatus e S. megalanthus e H. polyrhyzus (utilizando-se Hylocereus como doador de pólen) foi $100 \%$ equivalente aos cruzamentos interclonais de $S$. megalanthus e superior a autofecundação deste, que apresentou $60 \%$. O número de sementes produzidas por estes dois híbridos também foi superior (1407 e 1126 nos híbridos contra 891 nos cruzamentos interclonais e 508 em autofecundação de S. megalanthus). Tel-Zur et al. (2004) também analisaram clones de híbridos entre Hylocereus polyrhizus e Selenicereus megalanthus, e observaram tratar-se de triploides $(2 n=33)$ na maioria dos casos, embora também tenham registrado dois aneuploides $(2 n=34$ e 35$)$. As células-mãe do pólen (CMPs) exibiram na metáfase I univalentes, bivalentes e, ocasionalmente, quadrivalentes. A segregação anafásica mostrou anormalidades como fusos tripolares e foram observados tríades com frequência bem maior do que de díades. A viabilidade do pólen variou entre os quatro clones analisados de 9,8 a $18,6 \%$ e o diâmetro do pólen variou enormemente, provavelmente como resultado do número variável de cromossomos na sua composição. Apesar da origem alotriploide dos híbridos, gametas masculinos e femininos funcionais foram produzidos em proporções consideráveis, resultantes de uma segregação balanceada. Os clones triploides e aneuploides produziram sementes viáveis e a quantidade de sementes por fruto foi fortemente dependente do doador de pólen.

Parks e Boyle (2003) analisaram a fertilidade de sete clones de Schlumbergera, sendo dois diploides, dois triploides e três tetraploides, com base em viabilidade do pólen, produção de frutos e de sementes por fruto. Todos produziram grãos de pólen viáveis. Os diploides e um tetraploide foram autoincompatíveis, enquanto os demais poliploides foram autocompatíveis e produziram progênie quando autofecundados. Houve correlação positiva entre diâmetro do pólen e ploidia, sendo que nos diploides os grãos foram mais uniformes. Cruzamentos entre clones produziram sementes, sendo que o número maior foi encontrado entre clones diploides.

Mohanty et al. (1996) estudaram nove espécies de Mammillaria encontrando frequência variável de quiasmas (1,636 a 2,409 por bivalente) e esterilidade do pólen variando de 8,34 a $28,87 \%$. Nas duas espécies tetraploides observaram-se univalentes e trivalentes ou quadrivalentes e, consequentemente, as maiores taxas de esterilidade do pólen. No trabalho de Das et al. (1998b) a esterilidade encontrada em espécies de Mammillaria variou de modo semelhante, entre 9,02 a 26,46\%. Del Angel et al. (2006) também analisaram comportamento meiótico em espécies de Mammillaria e encontraram formação normal de bivalentes em $M$. dixanthocentron Backeb. ex Mottram. A frequência de quiasmas foi de $21,25 \%$ por célula e de 1,93\% por bivalente e os valores de viabilidade do pólen foram altos - acima de $90 \%$ - assim como no trabalho de Briones et al. (2004).

A formação de quadrivalentes foi observada também por Ross (1981) em três poliploides: Rebutia spegazziana Backeb., Rebutia sp. cv 'nivea' e Mammilaria prolifera. Outros poliploides analisados no trabalho formaram apenas bivalentes.

Baker e Pinkava (1987) estudaram o híbrido Opuntia $\times$ kelvinensis V.E.Grant \& K.A.Grant originado de Opuntia spinosior (Engelm.) Tourney e O. fulgida Engelm. e mostraram que quase todos os indivíduos investigados de O.×kelvinensis são triploides, que os indivíduos de Opuntia fulgida são, na maioria diploides, mas em parte triploides, e todos os indivíduos de O. spinosior são diploides. Todas as meioses de indivíduos diploides revelaram quase exclusivamente bivalentes, enquanto todas as meioses dos indivíduos triploides mostraram apenas trivalentes. A segregação desigual dos cromossomos nas células-mãe do grão de pólen durante a anáfase I provavelmente foi a causa da alta porcentagem de sementes estéreis observada no triplóide O.×kelvinensis.

\section{Considerações Finais}

Acredita-se que a família seja de origem relativamente recente (GIBSON e NOBEL, 1986; NYFFELER, 2002) e, talvez por isso, toda a família apresenta mesmo número básico com registros raros de disploidias e poucos rearranjos estruturais. Dessa maneira, uma alta homeologia cromossômica explicaria a frequente ocorrência de hibridizações interespecíficas. Por outro lado, a poliploidia é um importante fator evolutivo que gerando especiação. A ocorrência de reprodução vegetativa e apomixia facilitam, nos grupos em que ocorre, o estabelecimento destes poliploides. 


\section{Agradecimentos}

Os autores agradecem a Marlon Câmara Machado pela contribuição nas identificações taxonômicas; aos colegas Sheila Vitória Resende, Jamile Aquino, João Paulo Loyola pelo apoio técnico; à Fundação de Amparo à Pesquisa do Estado da Bahia - FAPESB (Projeto PRONEM T.O. 0020/2011) e ao BNB-FUNDECI Fundo de Desenvolvimento Científico e Tecnológico, pelo auxílio financeiro.

\section{Referências}

ANDERSON, E. F. The cactus family. Portland, Timber Press, Oxford, 2001. 776p.

APG III. An update of the Angiosperm Phylogeny Group classification for the orders and families of flowering plants: APG III. Botanical Journal of the Linnean Society, v.161, p.105-121, 2009.

APPLEQUIST, W. L.; WALLACE R. S. Deletions in the plastid trnT-trnL intergenic spacer define clades within Cactaceae subfamily Cactoideae. Plant Systematics and Evolution, v.231, p. 153-162, 2002.

ARAKAKI, M.; SOLTIS, D.E.; SPERANZA, P.New chromossome counts and evidence of polyploidy in Haageocereus and related genera in tribe Trichocereeae and other tribes of Cactaceae.Brittonia, v.59(3), p.290-297, 2007.

ASSIS, J. G. A.; OLIVEIRA, A. L. P. C.; RESENDE, S. V.; SENRA, J. F. V.; MACHADO, M. Chromosome numbers in Melocactus (Cactaceae). Bradleya, v.21, p.1-6. 2003.

BAKER, M. A.; PINKAVA, D. J.A cytological and morphometric analysis of a triploid apomict, Opuntia $\times$ kelvinensis (subgenus Cylindropuntia, Cactaceae).Brittonia, v.39(3), p.387-401, 1987.

BAKER, M. A.; REBMAN, J. P.; PARFITT, B. D.; PINKAVA, D. J.; ZIMMERMAN, A. D. Chromosome numbers in some cacti of western North America-VIII. Haseltonia, v.15, p.117-134, 2009.

BANDYOPADHYAY, B.; SHARMA, A.The use of multivariate analysis of karyotypes to determine relationships between species of Opuntia (Cactaceae). Caryologia, vol.53(2) p.121-126, 2000.

BARLOW, P. W.; NEVIN, D. Quantitative karyology of some species of Luzula.Plant Systematics and Evolution, v.125(2), p.77-86, 1976.

BARTHLOTT, W. Biogeography and evolution in neo- and paleotropical Rhipsalinae (Cactaceae).Verhandlungen des naturwissenschaftlichen Vereins in Hamburg, v.7, p.241-
$248,1983$.

BARTHLOTT, W.; HUNT, D.R. Cactaceae. In: KUBITZKI, K. (Ed.), The Families and Genera of Vascular Plants, vol. 2. Springer, Berlin, p.161-197, 1993.

BRIONES, F.; PALOMINO, G.; GARCIA, A. Chromosome analysis of Mammillaria supertexta, M. crucigera and M. haageana and their comparison with $M$. sanangelensis (Cactaceae).Caryologia, v.57(3), p.211-218, 2004.

CASTRO, J.P. Números cromossômicos em espécies de Cactaceae ocorrentes no nordeste do Brasil. 2008. $72 \mathrm{f}$. Dissertação (Mestre em Agronomia) - Universidade Federal da Paraíba, Areia.

CISNEROS, A.; GARCIA, R. B.; TEL-ZUR, N. Ovule morphology, embryogenesis and seed development in three Hylocereus species (Cactaceae).Flora, v.206, p.10761084, 2011.

COTA, J. H.; REBMAN, J.P.; WALLACE, R.S. Chromosome Numbers in Ferocactus (Cactaceae: Cactoideae). Cytologia, v.61, p.431-437, 1996.

COTA-SÁNCHEZ, J. H.; BOMFIM-PATRÍCIO, M. C. Seed morphology, polyploidy and the evolutionary history of the epiphytic cactus Rhipsalis baccifera (Cactaceae). Polibotanica, v.29, p.107-129, 2010.

CUÉNOUD , P.; SAVOLAINEN, V.; CHATROU , L. W.; POWELL, M. GRAYER, R. J.; CHASE, M.W. Molecular phylogenetics of Caryophyllales based on nuclear $18 \mathrm{~S}$ rDNA and plastid rbcL, atpB and matK DNA sequences. American Journal of Botany, v.89, p.132-144, 2002.

CROZIER, B.S. Subfamilies of Cactaceae Juss.including Blossfeldioideae subfam. nov. Phytologia, v.86(2), p.52-64, 2004.

DAS, A. B.; MOHANTY, S.; DAS, P. Variation in Karyotype and 4C DNA Content in Six Species of Melocactus of the Family Cactaceae.Cytologia, v.63, p.9-16, 1998a.

DAS, A. B.; MOHANTY, S.; DAS, P. Interspecific variation of nuclear DNA and structural changes in meiotic and mitotic chromosome in some species of Mammillaria (Cactaceae).Caryologia, v.51(3-4) p.289-301,1998b.

DAS, A. B.; MOHANTY, S.; DAS, P.Cytophotometric estimation of 4C DNA content and chromosome analysis in four species of Astrophytum Lem. of the family Cactaceae. Cytologia, v.65(2), p.141-148, 2000.

DAS, A. B.; MOHANTY, S. Karyotype analysis and in situ nuclear DNA Content in seven species of Echinopsis Zucc. of the Family Cactaceae. Cytologia, v.71(1), p.75-79, 2006. 
DEL ANGEL, C.; PALOMINO, G.; GARCIA, A.; MENDEZ, I. Nuclear size and karyotype in Mammillaria species (Cactaceae).Caryologia, v.59, p.177-186, 2006.

GIBSON, A. C.; NOBEL, P. S. The Cactus Primer. London, Harvard University Press, Cambridge, 1986. 286p.

GOLDBLATT, P. Index to plant chromosome numbers 19751978. Monographsin Systematic Botany from the Missouri Botanical Garden, v.5, 1981. 553p.

; Index to plant chromosome numbers 1979-1981. Monographsin Systematic Botany from the Missouri Botanical Garden, v.8, 1984. 427p.

; Index to plant chromosome numbers 1986-1987. Monographsin Systematic Botany from the Missouri Botanical Garden, v.30, 1990. 243p.

GORELICK, R. Resolving the phylogenetic placement of Blossfeldia liliputana (Cactaceae): Reticulate evolution, chloroplast inheritance, and graft-chimeras. Bradleya, v.22, p.9-14, 2004.

GRIFFITH, M. P. The origins of an important cactus crop, Opuntia ficus-indica (Cactaceae): new molecular evidence. American Journal of Botany, v.91(11), p.1915-1921, 2004.

GUERRA, M. S. Introdução à Citogenética Geral. Rio de Janeiro, Ed. Guanabara, 1988. 142 p.

HERNÁNDEZ-HERNÁNDEZ, T.; HERNÁNDEZ, H. M.; DE-NOVA, J. A.; PUENTE, R.; EGUIARTE, L. E., MAGALLÓN, S. Phylogenetic relationships and evolution of growth form in Cactaceae (Caryophyllales, Eudicotyledoneae). American Journal of Botany, v.98(1), p.44-61, 2011.

HUNT, D.R.; TAYLOR, N. P.; CHARLES, G. The New Cactus Lexicon.Milborne Port, DH Books, England, 2006. 925p.

JUDD, W.S.; CAMPBELL, C.S.; KELLOG, E.A.; STEVENS, P.F.; DONOGHUE, M.J. Plant Systematics.A phylogenetic approach.3a Ed. Sunderland, Sinauer Associates, 2007. 565 .

KIESLING, R. Origen, domesticación y distribución de Opuntia ficus-indica. Journal of the Professional Association for Cactus Development, v.3, 1998.Disponível em <http://www.jpacd.org/Jpacd98/kiesling.pdf >. Acesso em 09 jan. 2013.

LAS PEÑAS, M. L.; BERNARDELLO, G.; KIESLING, R. Karyotypes and fluorescent chromosome banding in Pyrrhocactus (Cactaceae).Plant Systematics and Evolution, v.272(1-4), p.211-222, 2008.
LAS PEÑAS, M. L.; URDAMPILLETA, J. D.; BERNARDELLO, G.; FORNI-MARTINS, E. R. Karyotypes, heterochromatin, and physical mapping of 18S-26S rDNA in Cactaceae. Cytogenetics and Genome Research, v.124, p.72-80, 2009.

LICHTENZVEIG, J.; ABBO, S.; NERD, A.; TEL-ZUR, N; MIZRAHI, Y. Cytology and mating systems in the climbing cacti Hylocereus and Selenicereus. American Journal of Botany,v.87(7), p.1058-1065, 2000.

LONE, A. B.; TAKAHASHI, L. S. A.;FARIA, R. T.;DESTRO, D. Desenvolvimento vegetativo de Melocactus bahiensis (Cactaceae) sob diferentes níveis de sombreamento. Revista Ceres, v.56(2), p.199-203, 2009.

MAJURE, L. C.; PUENTE, R.; PINKAVA, D. J. Miscellaneous chromosome numbers in Opuntieae DC.(Cactaceae) with a compilation of counts for the group.Haseltonia, v.18, p.67-78, 2012a.

MAJURE, L.C.; PUENTE, R.; GRIFFITH, M.P.; JUDD, W.S.; SOLTIS, P.S.; SOLTIS, D.S. Phylogeny of Opuntias.s. (Cactaceae): Clade delineation, geographic origins, and reticulate evolution.American Journal of Botany, v.99(5), p.847-864, 2012b.

MAJURE, L.C.; JUDD, W.S.; SOLTIS, P.S.; SOLTIS, D.S. Phylogeny of Opuntia s.s. (Cactaceae): Cytogeography of the Hemifusa clade of the Opuntia s.s. Mil.. 1754 (Cactaceae, Opuntioideae, Opuntieae): correlations with pleistocene refugia and morphological traits in a poliploid complex. Comparative Cytogenetics, v.6(1), p.53-77, 2012c.

MIHALTE, L.; SESTRAS, R.E.; FESZT, G.; TAMAS, E. Assessment of genetic variation on four genera of Cactaceae using taxonomic, cytological and molecular markers methods. Plant Omics Journal, v.4(3), p.142-148, 2011.

MOHANTY, S.; DAS, A.B.; DAS, P. Analysis of chiasma frequency and nuclear DNA variation in some species of Mammillaria.Cytobios, v.88, p.173-181, 1996.

MOHANTY, S.; DAS, A.B.; DAS, P. Studies on nuclear and meiotic chromosomes in 8 species on Mammillaria. Cytologia, v.62, p.331-336, 1997.

MOORE, R. J. Index To Plant Chromosome Numbers 19671971. Regnum Vegetabile,v.90, 1973, p.539.

NEGRÓN-ORTIZ, V. Reproductive biology of a rare cactus, Opuntia spinosissima (Cactaceae), in the Florida Keys: why is seed set very low? Sexual Plant Reproduction, v.11, p.208-212,1998.

NEGRON-ORTIZ, V. Chromosome numbers, nuclear DNA 
content, and polyploidy in Consolea, a endemic Cactus of Caribbian Island. American Journal of Botany, v.94(8), p.1360-1370, 2007.

NYFFELER, R. Phylogenetic relationships in the cactus family (Cactaceae) based on evidence from trnK/matK and trnL-trnF sequences. American Journal of Botany, v.89, p.312-326, 2002.

ORTEGA-BAES, P.; GOROSTIAGUE, P. Extremely reduced sexual reproduction in the clonal cactus Echinopsis thelegona. Plant Systematics and Evolution, v.299, p.785791, 2013.

ORTOLANI, F. A.; MATAQUEIRO, M. F.; MORO, J. R. Caracterização citogenética em Schlumbergera truncata (Haworth) Moran e Schlumbergera x buckleyi (T. Moore) Tjaden (Cactaceae). Acta Botanica Brasílica, v.21(2), p.361367, 2007.

PALOMINO, G.; DOLEZEL, J.; CID, R.; BRUNNER, I.; MÉNDEZ, I.; RUBLUO, A. Nuclear genome stability of Mammillaria san-angelensis (Cactaceae) regenerants induced by auxins in long-term in vitro culture. Plant Science, v.141, p.191-200, 1999.

PALOMINO, G.; HERAS, H. M. Karyotypic studies in Opuntia cochinera, O. hyptiacantha, and O. strepthacantha (Cactaceae).Caryologia,v.54(2), p.147-154, 2001.

PARKS, C.; BOYLE, T.H. Variation in ploidy level, fertility, and breeding behavior in cultivated Schlumbergera (Cactaceae).ISHS Acta Horticulturae 623:XXVI International Horticultural Congress, Plant Genetic Resources, The Fabric of Horticulture's Future. 2003.

PIERCE, B. Genética: um enfoque conceitual. Rio de Janeiro, Guanabara Koogan, 2004. p. 247-254.

PIMIENTA-BARRIOS, E.; MUÑOZ-URÍAS, A. Domesticação das opuntias e variedades cultivadas. In: Agroecologia: cultivo e usos de palma forrageira. SEBRAE/PB, 2001. p.58-63.

PINKAVA, D. J. On the evolution of the continental North American Opuntioideae.2002. In: SEGURA, S.; SCHEINVAR, L.; OLALDE, G.; LEBLANC, O.; FILARDO, S.; MURATALLA, A.; GALLEGOS, C.; FLORES, C. Genome sizes and ploidy levels in Mexican cactus pear species Opuntia (Tourn.) Mill. series Streptacanthae Britton et Rose, Leucotrichae DC., Heliabravoanae Scheinvar and Robustae Britton et Rose. Genetics Resources and Crop Evolution, v.54.p.1033-1041, 2007.

PINKAVA, D. J.; McLEOD, M. G.; McGILL, L. A.; BROWN, R. C. Cromosome numbers in some cacti of western North America. II. Brittonia, v.25, p.2-9, 1973.
PINKAVA, D. J.; REBMAN, J.B.; BAKER, M.A. Cromosome numbers in some cacti of western North America - VII. Haseltonia, v.6, p.32-40, 1998.

POWELL, A. M.; WEEDIN, J. F. Chromosome numbers in Chihuahuan desert Cactaceae. III. Trans-Pecos Texas. American Journal of Botany, v.88(3), p.481-485, 2001.

ROSS, R. Chromosome counts, cytology, and reproduction in the Cactaceae. American Journal of Botany, v.68, p.463470, 1981.

SAHLEY, C. T. Bat and Hummingbird Pollination of an Autotetraploid Columnar Cactus, Weberbauerocereus weberbaueri (Cactaceae). American Journal of Botany, v.83(10), p.1329-1336, 1996.

SCHLUMBERGER, B.; RENNER, S.S. Molecular phylogenetics of Echinopsis (Cactaceae): polyphyly at all levels and convergent evolution of pollination modes and growth forms. American Journal of Botany, v.99(8), p.1335-1349, 2012.

SEGURA, S.; SCHEINVAR, L.; OLALDE, G.; LEBLANC, O.; FILARDO, S.; MURATALLA, A.; GALLEGOS, C.; FLORES, C. Genome sizes and ploidy levels in Mexican cactus pear species Opuntia (Tourn.) Mill. Series Streptacanthae Britton et Rose, Leucatrichae DC., Heliabravoanae Scheinvar and Robustae Britton et Rose. Genetics Resources and Crop Evolution, v.54, p.1033-1041, 2007.

STOCKWELL, P. Chromosome numbers of some the Cactaceae.Botanical Gazette, v.96, p.565-570, 1935.

TAYLOR, N. P. Taxonomy and Phytogeography of the Cactaceae of eastern Brazil.2000, 414f.Tese ('Doctor of Philosophy') - Royal Botanic Garden, Kew.

TEL-ZUR, N.; ABBO, S.; MIZRAHI, Y.Cytogenetics of semi-fertile triploid and aneuploid intergeneric vine cacti hybrids. Journal of Heredity, v.96(2), p.124-131, 2004.

WEEDIN, J.F.; POWELL, A.M.IOPB chromosome number reports LX. Taxon, v.27, p.223-231, 1978. 